\title{
Commentaries
}

\section{Telemedicine and computers in diabetic retinopathy screening}

Providing full screening of diabetic patients for retinopathy throughout the community would significantly reduce the incidence of blindness in this group of patients. However, although panretinal photocoagulation is of proved value in reversing or preventing neovascular complications and careful macular treatment prevents further visual loss, the resources or the infrastructure to detect appropriate patients for treatment are not universally available. ${ }^{1}$ The importance of the situation has been highlighted by the declaration of St Vincent, a European directive on the need to provide screening. ${ }^{23}$ An effective screening and treatment programme would ultimately reduce the burden on health and social service budgets because fewer of these relatively young patients would require long term support. ${ }^{4-6}$

Locally based efforts indicate that the key to diabetic retinopathy screening is efficiently obtaining images of the retina for classification. This raises two problems - firstly, the target population must be reached at minimum inconvenience to the patient and, secondly, the images must be assessed effectively. Care facilities for diabetic patients are spread throughout the community and so it is most appropriate that their retinas be examined there. Unfortunately, the expertise for assessment of diabetic retinopathy is not always available locally. Therefore systems involving fundus photography with later assessment of the image by a specialist have been employed but these use relatively expensive photographic prints, slides, or Polaroids with inefficient manual transport of images.

The technological revolution has provided us with new methodologies that we should exploit to solve these problems. For example, it is now possible to digitise images and send these through telemedicine-for example, email, with the advantages of computerised storage and image manipulation. Although commercial fundus cameras can be used to obtain and store digitised image data their database, storage systems do not easily lend themselves to data analysis (a problem that the companies involved should address otherwise investigators will be forced to design their own systems). Digital cameras are available and are becoming cheaper. Also, competition in the market for scanning laser ophthalmoscopes is increasing. Cost savings are likely if the cameras can be placed in the community ${ }^{7}$ with transfer of the digitised images through various methods. Compression of the images allows faster data transfer and reduces storage requirements but the methods are diverse requiring investigators to decide carefully the best ones. This is a difficult area because as anyone who has bought the latest PC computer knows your "pride and joy" is out of date within a few years such is the speed of development of computer technologies. The data must also be confidential and so not all methods of data transfer are appropriate.

Potentially, digitised data can be analysed by computer either at the site of acquisition of the image or after transfer to a centralised "sorting" area. If the images are processed by computer the need for assessment by technical or ophthalmological staff is removed with associated reductions in cost. ${ }^{8}$ The ultimate goal would involve automated acquisition of the photographic image, digitisation, and analysis without any intervention by technical staff.
The computer would then indicate to the patients that they require to attend an ophthalmology department. Currently, artificial neural network (ANN) computer programs are capable of discriminating normal fundi from those with retinopathy, potentially reducing the numbers of images requiring expert examination by $70 \%$ or more. ${ }^{9} 10$ The application of such programs in the field has yet to be assessed but the rapid progress in PC technology is likely to increase the ability to diagnose automatically fundus images. Continued development of ANN programs is likely to be a fruitful area of research especially if the tasks are relatively simple-for example, whether diabetic retinopathy is present or not. More complex image analysis such as grading of retinopathy into background, preproliferative, and proliferative will be much more difficult to achieve. New methods involving parallel ANNs, ${ }^{11}$ real time "on chip" video signal processing, ${ }^{12}$ or the use of workstation computers may help.

Although services for diabetic screening are fragmented existing facilities could be adapted to digitising methods with relatively small initial investment. Pilot schemes should be commenced investigating the most appropriate technologies available while allowing some adaptability for any new developments that may occur. Already, departments in Europe are involved in this area of research. These should be brought together to share their experiences to date, to reduce duplication of effort, and, hopefully, to produce a coordinated approach to the problem. If digitisation of diabetic images proves practical, as is likely, investment on a national scale may be necessary to produce the infrastructure required. Remember that care of diabetic patients already consumes approximately $8 \%$ of the UK national healthcare budget. ${ }^{13}$ Effective computerised screening could potentially reduce that burden while bringing major benefits to patients.

Merely browsing the technical sections of the broadsheet newspapers shows how fast digital cameras, data storage, and data transfer are progressing, largely fuelled by the needs of the video and music industry. Progress is relentless and provides us with opportunities to solve practical problems in the management of our patients. Surely on the back of such developments we will be able to find a solution for a logistical problem such as diabetic retinopathy screening.

Supported by British Diabetic Association Grant No x88202.

T H WILLIAMSON

Department of Ophthalmology, St Thomas's Hospital, Lambeth Palace Road, London SE1 7EH

D KEATING

Department of Clinical Physics and Bio-Engineering, and Tennen Institute of Ophthalmology, Western Infirmary, 38 Church Street, Glasgow G11 6NT

1 Rohan TE, Frost CD, Wald NJ. Prevention of blindness by screening for diabetic retinopathy: a quantitative assessment. $B M \mathcal{F}$ 1989;299:1198-201. Diabetes care and research in Europe: the St Vincent Declaration. Diabet Med 1990;7:360.

3 British Diabetic Association. St Vincent joint task force for diabetes. Final report. London: BDA, 1995 .

4 Sculpher MJ, Buxton MJ, Ferguson BA, Spiegelhalter DJ, Kirby AJ. Screening for diabetic retinopathy:a relative cost-effectiveness analysis of alterna-
ind tive modalities and strategies. Health Econ 1992;1:39-51. 
5 Javitt JC, Canner JK, Frank RG, Steinwachs DM, Sommer A. Detecting and reating retinopathy in patients with type I diabetes mellitus. A health policy model. Ophthalmology 1990;97:483-94.

6 Javitt JC, Canner JK, Sommer A. Cost effectiveness of current approaches to the control of retinopathy in type I diabetics. Ophthalmology 1989;96:255-64.

7 Sculpher MJ, Buxton MJ, Ferguson BA, Humphreys JE, Altman JF, Spiegelhalter DJ, et al. A relative cost-effectiveness analysis of different methods of screening for diabetic retinopathy. Diabetic Med 1991;8:644-50.

8 Lairson DR, Pugh JA, Kapadia AS, Lorimor RJ, Jacobson J, Velez R. Costeffectiveness of alternative methods for diabetic retinopathy screening. Diabetes Care 1992;15:1369-77.

9 Gardner G, Keating D, Williamson TH, Elliot AT. Automatic detection of diabetic retinopathy using an artificial neural network: a screening tool. $\mathrm{Br}$ f Ophthalmol 1996;80:940-4.
10 Gardner G, Keating D, Williamson TH, Elliot AT. Detection of diabetic retinopathy using neural network analysis of fundus images. Proceedings of International Conference on Neural Networks and Expert Systems in Medicine and Health Care. University of Plymouth, 1996:2835 .

11 Kerr JP, Bartlett EB. Medical image processing utilizing neural networks trained on a massively parallel computer. Comput Biol Med 1995;25:393403.

12 Naylor D, Jones S, Myers D, Vincent J. Neural network feature detector for real-time video signal processing. Int $\mathcal{F}$ Neural Syst 1993;4:337-49.

13 King's Fund Policy Institute. Counting the cost: the real impact of non-insulin dependent diabetes. London: British Diabetic Association, 1996.

\section{How dangerous a world is it?}

In the Western world approximately $2-3 \%$ of all pregnancies result in an infant with a major malformation. Inevitably the parents of such children undergo a period of grieving for "the loss of their normal child" and as a part of that grief they seek to apportion blame. If ocular involvement is a feature of the malformation then it is during the period of their grief that we as ophthalmologists are likely to come into contact with the family. We need to understand this process and during our early contact with the family avoid the twin problems of either being dismissive of their search for a causative agent or, alternatively, being too eager to support their proposal that a particular action or event has been responsible for the outcome of the pregnancy. We are rarely aided in this by the attentions of the "popular press" where the demands of investigative journalism are incompatible with a studied evaluation of possible cause and effect. When trying to identify the effect of a possible teratogen on a pregnancy, there are well established criteria which help in the attribution of blame.

The possible association is often first recognised through case reporting (and that remains a major value of the isolated case report), but these can be misleading. The picture of affected pregnancies in cocaine addicted mothers is a case in point, where it begins to seem possible that some of the described abnormalities are a consequence of other features of the addict's lifestyle, such as poor nutrition and alcohol abuse, rather than the cocaine itself. It is important, therefore, that epidemiological studies should be undertaken to demonstrate a statistical association between exposure to the "teratogen" and an abnormality in exposed children (ideally in different population groups). The thalidomide tragedy of the late 1950 s and early '60s demonstrated this mechanism, but thalidomide was remarkable in that as far as can be determined $20 \%$ to $25 \%$ of all exposed pregnancies resulted in a child with major malformations, while exposure between the 20th and 40th day after conception always resulted in some limb shortening. ${ }^{1}$ Few if any other teratogens have had such a "strike rate" (which allowed relatively rapid identification with epidemiological studies in Scandinavia particularly), so for other agents identifying an association may be more delayed.

Corroborating evidence can be derived from longitudinal studies if they are able to show an increase in a particular defect associated with exposure to the teratogen, accompanied by a reduction when the teratogen is eliminated. Again, this approach has been valuable with the rubella virus, identifying first a high incidence of the profound embryopathy with first trimester exposure, and then subsequently a reduction in frequency following the introduction of effective immunisation.
Animal studies showing a teratogenic effect from the agent being investigated are useful though subject to the vagaries of species variation, and it is also helpful to look at the dynamics of an agent under suspicion to see if its mode of action (in the case of a chemical) shows the potential to interfere with organogenesis (as might be expected if the pregnant mother is using antimetabolites). These criteria have been enunciated by Brent ${ }^{2}$ and serve as guidelines when we as clinicians evaluate reports of induced ocular malformations.

The danger of accepting unsubstantiated associations was demonstrated in 1980 when major defects, including cyclopia, were attributed to salicylate ingestion during the first trimester. ${ }^{3}$ Only a properly conducted, prospective, collaborative study was able to allay the anxiety induced by these reports. However, we must at the same time guard against an over-resistance to information coming to us from non-medical sources, since we then run the risk of failing to identify a teratogen and expose further pregnancies unnecessarily.

This dilemma has recently been highlighted by the publicity surrounding the benzimidazole antifungal agent Benomyl. In January 1993 under the headline "Mystery of babies with no eyes" an article appeared in The Observer detailing the birth of nine children in an area of Lincolnshire within a 40 mile radius of Louth village, born with microphthalmia or anophthalmia. The report went on to identify other clusters in the UK, some, though by no means all, occurring in areas where agriculture (and thus the possible use of a popular fungicide) represented a major feature of the economy. The report cited evidence from animal experiments carried out in 1991 in which craniocerebral defects, including ocular malformation with retinal dysplasia and microphthalmos/anophthalmos, occurred in the offspring of pregnant rats fed a dose of Benomyl of $62.4 \mathrm{mg} / \mathrm{kg}$ between the 7 th and $21 \mathrm{st}$ days of the pregnancy. ${ }^{4}$ The researchers speculated that the antitubulin action of Benomyl could conceivably impair microtubule formation and thus disrupt neuronal proliferation and migration.

The leap from this preliminary animal work (which could not be reproduced in a rabbit model) to ascribing isolated microphthalmos with no associated malformation to possible exposure to the fungicide being used in an agricultural or market garden setting is enormous. Nevertheless, in June 1996 the American courts awarded US $\$ 4$ million to the Castillo family on the basis that the microphthalmos affecting their child might be caused by exposure to Benomyl. The child had no other neurological deficit and was not growth retarded. 
Subsequently, the support group in the United Kingdom have, naturally, seized upon this judgment, and many of us have met parents who now confidently accept the association. What then are we to do? To dismiss this legal decision would be naive, even though the evidence presented in court was heavily criticised. What seems most important is to have a mechanism which can rapidly and effectively investigate this and any future worries, using the principles which Brent has laid down. Yet again this episode indicates the pressing need for a continuation of our move towards "evidence based" medicine, but in addition it suggests a role for a rapid response mechanism when a possible toxic effect is suggested, so that the popular press with its different priorities, is not left to take the lead. The current reporting system should work when medicines come under the spotlight, but is inappropriate for environmental agents. Perhaps the Royal College of Ophthalmologists, in conjunction with its sister colleges, might consider the establishment of a body to scan the scientific literature, and ensure that the clinicians in daily contact with patients are kept as informed as possible.
For the time being the case against Benomyl continues to be investigated. Since Australia banned its use several years ago it might well be time for the Australian College of Ophthalmologists to assess whether the incidence of this particular malformation has fallen in their community. In the United Kingdom isolated reports continue to appear, sometimes associated with very high dose application to nearby crop growing areas, and coordination of this reporting is available.

H E WILLSHAW

Children's Hospital,

Ladywood, Birmingham

1 Lentz W. A short history of thalidomide embryopathy. Teratology 1988;38:203.

2 Brent RL. Methods of evaluating the alleged teratogenicity of environmental agents. Teratogen Update 1986;199-201.

3 Benawra R, Mangunten H H, Duffell DR. Cyclopia and other anomalies following maternal ingestion of salicylates. F Pediatr 1984;96:1069.

4 Hoogenboom ER, Randsdell JF, Ellis WE, Kavlock RJ, Zeman FJ. Effects on the fetal rat eye of maternal benomyl exposure and protein malnutrition. Curr Eye Res 1991;10:601-12. 